\title{
La relación entre seguridad e inmigración durante las primeras décadas del siglo XX en Argentina
}

\author{
Andrés Pereira \\ Centro de Estudios Avanzados, Universidad Nacional de Córdoba. \\ Email: pereiramanuelandres@gmail.com
}

\begin{abstract}
Resumen $^{1}$ : El presente artículo tiene como objetivo comprender la forma en que se configuró la relación entre seguridad e inmigración durante las primeras décadas del siglo XX en Argentina. De esta manera, el texto busca contribuir a rellenar la brecha que existe entre el temprano interés que el Estado argentino ha demostrado en relación al delito, la seguridad y la inmigración, y la escasa indagación específica sobre estos temas que existe en los estudios migratorios de Argentina. Esto se llevará adelante a través del análisis de diferentes documentos estatales, en particular leyes, decretos, fallos judiciales y de la revisión de un conjunto de textos que han abordado diferentes aspectos de la relación entre Estado e inmigración durante el período seleccionado. El abordaje teórico articula el trabajo de Abdelmalek Sayad junto con los desarrollos en relación al vínculo entre seguridad y migración hechos por los estudios críticos de la seguridad.
\end{abstract}

Palabras clave: inmigración, securitización, pensamiento de Estado, Argentina.

\section{The relationship between security and immigration during the early twentieth century in Argentina}

\begin{abstract}
This article aims to understand how the relationship between security and immigration is set during the early twentieth century in Argentina. Thus, the text seeks to help fill the gap between the early interest that the Argentine state has shown in relation to the crime, security and immigration, and the scarce specific inquiry on these issues that exists in migration studies Argentina. This will be carried out through the analysis of various state documents, including laws and decrees, and the review of a set of texts that have addressed different aspects of the relationship between state and immigration during the selected period. The theoretical approach articulates Abdelmalek Sayad's work together with the developments in relation to the connection between security and migration made by critical security studies.
\end{abstract}

Keywords: migration; securitization; state thinking; Argentina.

\section{A relação entre segurança e imigração durante as primeiras décadas do século XX na Argentina}

Resumo: Este artigo tem como objetivo compreender como se desenvolveua relação entre a segurançaeaimigraçãodurante as primeiras décadas do século XX, na 
Argentina. Assim, o texto procura ajudar a preencher a lacuna entre o interesse inicial que o Estado argentino temdemonstradoemrelaçãoaocrime, segurança e imigração, ea escassapreocupação específica sobre estas questõesque existe nos estudos de migração Argentina. Isto será realizado através da análise de diversos documentos estatais, incluindoleis, decretos, decisõesjudiciais e a revisão de um conjunto de textos que abordaram diferentes aspectos da relação entre Estado e aimigração durante o período selecionado. A abordagem teórica articula o trabalho de Abdelmalek Sayad, juntamente com os desenvolvimentosemrelação à ligação entre segurança e migraçãofeita pelosestudos críticos de segurança.

Palavras-chave: imigração; securitização; pensamento do Estado; Argentina.

$$
* * *
$$

\section{Introducción}

El presente artículo tiene como objetivo comprender la forma en que se desarrolló la relación entre seguridad e inmigración durante las primeras décadas del siglo XX en Argentina. Las investigaciones en el campo de los estudios migratorios en Argentina, no han abordado como objeto de indagación específico la relación entre seguridad y migración. En el contexto local, las cuestiones relativas a esta temática sólo han sido exploradas de modo subordinado a otros enfoques más generales como el análisis de las políticas migratorias.Una excepción, son los trabajos que han analizado la construcción del "inmigrante indocumentado" como delincuente, en los discursos de la Policía Federal y el discurso mediático, durante la década de 1990 (GEADIS, 2002; Pacecca, Courtis y Pita 2009). Por otra parte, desde disciplinas como la criminología crítica y la historia, investigaciones más recientes han tratado temáticas tales como la conformaciónde las conferencias sudamericanas de policía y la problemática de los delincuentes viajerosa principios del siglo XX (Galeano, 2009), y el surgimiento de las técnicas de identificación entre finales del siglo XIX y principios del XX (García, 2010; 2015). Aunque no han abordado de modo específico el nexo entre seguridad y migración, brindan nuevos elementos para comprender ciertas prácticas que se encuentran en el corazón de dicha relación.

La perspectiva teórico-metodológica que propongo se construye a partir de una apropiación y reformulación del concepto de securitización o (in)securitización ${ }^{2}$ de las migraciones (Bigo, 2002; Bigo y Tsoukala; 2008) articulado a claves teóricas como las de pensamiento de Estado, doble pena y expulsabilidad (Sayad, 2010; Sayad, 2008). De este modo, retomo una idea ampliamente difundida entre los académicosde la escuela de Paris, que inspirados en buena medida por teóricos como Michel Foucault y Pierre Bourdieu, han sostenido que el proceso de securitización de las migraciones debe ser interpretado no sólo a partir de los discursos sino también de las prácticas que ejercen diversos actores(Bigo, 2002; Huysmans, 2006; Huysmans y Squire, 2009). La escuela de París ${ }^{3}$, junto con las de Copenhague y Aberystwyth forma parte de los estudios críticos de la seguridady se distinguen de la literatura hegemónica sobre seguridad orientadas por enfoques neorrealistas y neoliberales (Bigo, 2008). 
En concordancia con la perspectiva teórico-metodológica adoptada, el análisis que utilizo es de tipo cualitativo y tiene como sustento empírico un conjunto de documentos, entre los que se pueden encontrar leyes, decretos, resoluciones, e informes de fuerzas de seguridad, así como también fallos de la Corte Suprema de Justicia de la Nación (CSJN). Las fuentes utilizadas a lo largo del trabajo fueron seleccionadas debido a que permiten entender el rol relevante que ocupan las prácticas administrativas en la relación seguridad einmigración. Los documentos legislativos me han permitido acercarme a los discursos de las élites, mientras que los decretos, resoluciones, informes y fallos judiciales posibilitanla visualización de las prácticas administrativas:diferencias, continuidades y contradicciones que emergen entre los discursos y prácticas sólo pueden ser analizadas a partir de esta variedad de documentos que permiten comprender el Estado sin concebirlo como una unidad homogénea. La utilización de bibliografía secundaria, ha sido de utilidad para contextualizar, apoyar y profundizar las reflexiones desplegadas a lo largo del texto, como así también para abrir interrogantes.

A lo largo de los apartados intento mostrar la relevancia que adquieren ciertas prácticas y técnicas administrativas en el proceso de producción de la inmigración como un problema de seguridad. De esta forma, planteo una respuesta a la pregunta sobre la relevancia que adquieren de los actores burocráticos en la producción de la (in)securitización las migraciones. No propongo restar relevancia a los discursos de las élites y al discurso jurídico desplegado en la normativa migratoria: lo que señalo es que las prácticas y técnicas no son el resultado de una securitización producida en primera instancia por unos discursos, sino que son una parte central y constitutiva del proceso y no un mero reflejo o epifenómeno. Además, en ese contexto, muestro algunos elementos que nos permiten pensar que las primeras décadas del siglo XX, no sólo significaron la securitización de las migraciones, sino también de las movilidades en términos más generales. En última instancia, y a modo de sugerencia, afirmo que es necesario indagar la forma en que los discursos y prácticas que producen la (in)securitización se constituyen a partir de la circulación transnacional de saberes e instituciones.

Para cumplir con los objetivos,he dedicado un primer apartado a exponer las opciones teóricas y metodológicas para abordar el proceso de securitización de las migraciones; en una segunda sección analizo la relevancia del anarquismo, en la constitución de discursos y prácticas que loconvierten en una amenaza para el orden social establecido; en una tercera parte brindo algunos elementos para comprender cómo, a partir de las prácticas de la Dirección General de Inmigración (DGI), convalidadas por la CSJN y la normativa migratoria posterior, se expande el nexo entre seguridad y migración hacia todo tipo de movilidades; en las conclusiones realizo un repaso descriptivo por las tesis más relevantes del artículo y esbozoalgunas sugerencias para el desarrollo defuturas investigaciones. 


\section{Algunas consideraciones teóricas sobre el nexo entre seguridad y migración}

Los estudios actuales sobre la securitización o (in)securitización de las migraciones, han contribuido de manera decisiva a la comprensión del modo en que las migraciones son tratadas de modo creciente como una cuestión de seguridad. Orientados al análisis de un conjuntode fenómenos que se despliegan a partir de las últimas décadas del siglo XX, los primeros estudios que acuñaron la noción de securitización insistieron en la relevancia de los actos de habla (speech acts) como modo de comprender la securitización de las migraciones (Weaver, 1998; Weaver, Buzan y Kelstrupr, et.al, 1993). Desde estas perspectivas, centradas al análisis discursivo, las explicaciones de por qué las migraciones internacionales se convirtieron en una cuestión de seguridad, se reducían a la capacidad performativa de ciertos actores estatales o no estatales. Estas explicaciones fueron criticadas más tarde por diversos académicos que comprendían que la securitización de las migraciones no podía quedar reducida al aspecto lingüístico sino que también debería comprender un conjunto de prácticas ejecutadas por diversos actores (Bigo, 2002; Huysmans y Squire, 2009; Walters, 2010).

En el marco de estas discusiones e intercambios entre académicos, se ha definido a la securitización de las migraciones como un proceso que excede el resultado de la propaganda de los partidos políticos de la extrema derecha o la efectividad creciente de retóricas que visualizan a algunas poblaciones como un peligro (Bigo, 2002). De este modo, a la hora de definir la (in)securitización de la inmigración como un riesgo,no sólo se tienen en cuenta los actos de habla exitosos que provienen de actores estatales o no estatales, sino que se ven como el resultado de discursos y prácticas estructuradas por el habitus de los profesionales de la seguridad que se mueven en un campo crecientemente organizado de modos transnacional (Bigo, 2002; Bigo y Tsoukala, 2010). Entre las prácticas que definen el concepto se destacan las administrativas, tales como la creación de perfiles de población, la evaluación de riesgos, las estadísticas o la creación de categorías (Bigo, 2002).

Es evidente que sería cuando menos un anacronismo intentar explicar la securitización de las migraciones, en Argentina a principios del siglo XX, "aplicando" esta perspectiva teórica que ha sido originada para comprender las sociedades de riesgo neoliberales y, en particular, en el contexto político de la Unión Europea. Sin embargo, esto no impide que retome algunos de sus postulados y los reformule o adapte en una definición más próxima al contexto argentino de principios del siglo XX. En este sentido, aquí defino a la securitización o (in)securitización, retomando la perspectiva de Bigo, como un conjunto de discursos y prácticas, configurados por el pensamiento de Estado, que producen a la inmigración como una amenaza para el orden social establecido por las élites entren finales del siglo XIX y el XX. La adaptación de este concepto no busca reemplazar las propuestas que han renovado la interpretación de la securitización más allá de los actos 
de habla, por el contrario, busca jugar con el concepto de securitización en otros contextos y enriquecerlo a partir de su redefinición contextualizada.

A partir de finales del siglo XIX, pero sobre todo desde principios del siglo XX, una porción de la inmigración europea, comenzó a ser comprendida en el discursojurídico, y también en el de las élites locales, como una amenaza a la "seguridad nacional” y al "orden público”. La noción de "seguridad nacional” servía para indicar que este grupo de inmigrantes, especialmente los identificados como "anarquistas", constituían una peligro parael orden social, político o económico construido e imaginado por las élites locales; la idea de "delito" se encontraba estrechamente relacionada a la de orden público ya que se utilizaba para designar en buena medida el crimen político o contra las instituciones del Estado pero también al delito común; la noción de "orden público" se refería a las conductas reguladas por los edictos policiales y los códigos contravencionales, marcados por una fuerte impronta positivista (Tiscornia, 2004a; 2004b). Estas expresiones jurídicas, producían y al mismo tiempo justificaban un tratamiento especial para los migrantes considerados como una amenaza para el orden social. A partir de la definición brindada en los párrafos anteriores es relevante y necesario comprender que este discurso iba acompañado de un conjunto de prácticas administrativas sin las cuales no se puede comprender de modo acabado la (in)securitización de las migraciones.

En este sentido, es imprescindible visibilizar que en paralelo al despliegue de estas nociones,que representaban una porción de la inmigración como amenaza, a medida que avanzó el siglo XX se fueron desplegando algunas prácticas y técnicas que no son el resultado de la (in)securitización sino que la posibilitan y la constituyen como tal. Entre ellas, se puede mencionar el desarrollo de los métodos de identificación de individuos desarrollados principalmente por las fuerzas de seguridad, tales como la identificación fotográfica, la antropometría y la dactiloscopia(García,2010; 2015); la incorporación de solicitudes de documentación que permitiera acreditar los antecedentes penales de los individuos, dirigidas en un primer momento a quienes ingresaban al país en la categoría de "inmigrantes" y ampliada posteriormente para todo tipo de movilidades; la emergencia de prácticas de cooperación o intercambio de información entre las diferentes instituciones se seguridad al interior del país como con otros países sudamericanos y europeos (Galeano, 2009). Así, no son sólo los discursos de las élites los que abren el proceso de (in)securitización de las migraciones, sino que se produce una articulación de discursos y prácticas que legitiman un modo de comprender la inmigración como una amenaza al orden social.

La transformación de la inmigración como una amenaza y el despliegue de un conjunto de medidas orientadas hacia el control de la migración, muestran que el orden social, vinculado al interés de las élites por la inserción de Argentina en el mercado internacional y el desarrollo capitalista, se encontraba atravesado por una dimensión nacional. La noción de pensamiento de Estado entendida como un conjunto de principios de visión y división del mundo social producidos y avalados por el Estado (Bourdieu, 
1997), que en relación a las migraciones se conforman como categorías nacionales y nacionalistas de pensamiento (Sayad, 2010), permite visualizar la especificidad que acarrea la relación entre seguridad y migración. Si bien en las primeras décadas del siglo XX los “criminales” o las denominadas “clases peligrosos” se convierten a en una cuestión de seguridad, la inmigración como asunto securitizado revela algunas dimensiones particulares.

Siguiendo esta línea, se puede afirmar que el pensamiento de Estado, y por lo tanto la distinción entre nacionales y no nacionales (Sayad, 2008) es constitutiva del Estado-nación: pues resulta consustancial para el Estado dotarse "de todos los criterios de pertinencia necesarios para proceder a esta discriminación, sin las que no hay Estado nacional (Sayad, 2010: 387). De esta manera, la (in)securitización de las migraciones, refleja y refuerza el tratamiento diferenciado entre aquellos sujetos considerados como nacionales o no nacionales. Esto se ve con claridad, por ejemplo, en relación a las expulsiones, ya que mientras los inmigrantes como no nacionales se transforman en sujetos expulsables ${ }^{4}$, a los que se le aplica una doble ${ }^{5}$ pena (Sayad, 2010), los individuos nacionales ingresan al sistema penal pero no pueden ser expulsados. Así, se puede analizar, cómo las prácticas llevadas adelante por las instituciones encargadas del control migratorio se encuentran conformadas a partir de las categorías naturalizadas por actores que contribuyen a su consolidación.

\section{La (in)securitización de la inmigración europea entre finales del siglo XIX y principios del XX}

En el período de tiempo seleccionado, la noción de inmigración, tanto en los discursos sociales como jurídicos, adquirió un significado muy específico. Entre mediados del siglo XIX y principios del siglo XX, la definición de inmigrante cristalizada en la normativa jurídica, se constituyó principalmente por las definiciones en la Constitución Nacional de 1853 y el la Ley $N^{\circ} 817^{6}$ de inmigración y colonización del año 1876. Puede decirse que esta visión definía a la "inmigración” como europea, civilizadora, y vinculada principalmente al trabajo agrícola, aunque con un sentido más amplio también recogía otras categorías ocupacionales como las de jornaleros, artesanos, industriales y profesores (Devoto, 2009). La europea, aparte de ser una inmigración fomentada, era visibilizada como una pieza clave para el desarrollo de la economía capitalista y la inserción de la argentina en el mercado mundial (Novick, 1997).

Por otro parte, algunos puntos de contacto entre seguridad y migración ya se podían encontrar en la Ley $N^{\circ} 817$. La norma, prohibía a los buques de pasajeros trasladar en calidad de inmigrantes a "enfermos de mal contagioso o de cualquier vicio orgánico que los haga inútiles para el trabajo, ni dementes, mendigos, presidiarios o criminales que hubiesen estado bajo la acción de la justicia”. La mención a los “presidiarios” y "criminales” da cuenta la existencia de una preocupación por el delito que en esa fecha 
todavía no se relacionaba con las acciones que se atribuirán posteriormente al anarquismo como por ejemplo los atentados o la colocación de artefactos explosivos (Scarzanella, 2003). Asimismo, la exclusión de los "presidiarios” y “criminales” en la categoría de inmigración respondía centralmente a la idea instrumental que existió alrededor de ésta, pues si “el criterio que definía al criminal era su incapacidad para dedicarse e identificarse con un trabajo regular” (Gayol y Kessler, 2002: 17; Cf. Ruibal, 1993) difícilmente podría incluirse a éste dentro de la categoría de inmigración, pensada en primer lugar en relación al trabajo agrícola.

Por otra parte, es relevante señalar algo que no ha sido lo suficientemente destacado, y es que la Ley $\mathrm{N}^{\circ} 817$ ya establecía que la acreditación de las condiciones para ser considerado inmigrante se validarían "por medio de certificados de los Cónsules o Agentes de inmigración de la República en el Exterior", o en su defecto "por certificados de las autoridades del domicilio del inmigrante, legalizados por los referidos Cónsules o Agentes de Inmigración de la República”. En este sentido, se puede observarde modo temprano, que los certificados y registros producidos por el Estado se utilizaban para distinguir la inmigración "viciosa e inútil” de la "honorable y laboriosa” según se definían en la ley. Con el transcurso de los años, las normas que reglamentaron la Ley de migraciones especificaron y renovaron periódicamente los certificados que se solicitaban y las características que debían tener para ser considerados válidos. Siguiendo a Torpey (2000), se puede afirmar que la solicitud de los diferentes documentos, pasaportes, tarjetas de identificación o certificados, junto con la creación de registros y archivos, permitió al Estado identificar y seleccionar a los beneficiarios de sus políticas, como así también a los individuos que se deberían ser excluidos de ellas.

En este sentido, la definición de las amenazas, y en particular de los sujetos que las encarnan, no sólo dependía de las representaciones alrededor de los inmigrantes, sino también de las técnicas y modalidades que permitieran hacer esas distinciones y plasmarlas en papeles y registros. Parafraseando a Torpey, si la distinción entre nacionales y extranjeros sólo es posible con el pasaporte, la diferenciación entre criminales y no criminales, también dependerá de los documentos y registros disponibles en la época. De todos modos, en este primer momento, las consideraciones alrededor de los criminales o presidiarios en la legislación migratoria, así como las técnicas de identificación y registro, no implican una securitización de la inmigración, pues si bienes posible hallar algunos nexos entre seguridad y migración, la inmigración europea aún no es considerada como una amenaza. Aunque ya existen algunas prácticas administrativas y técnicas, que servirán posteriormente al proceso se (in)securitización, éste cobrará forma y contenido con la emergencia de la cuestión social y el anarquismo.

Entre finales del siglo XIX y principios del siglo XX, el país experimentó un conjunto de rápidas transformaciones económicas, políticas y demográficas que crearon nuevos problemas y desajustes al proyecto propuesto por las élites gobernantes (Ruibal, 1993). En este contexto, las malas 
condiciones de trabajo, salud, alimentación y vivienda que padecían numerosos sectores de la población, muchos de ellos extranjeros, propiciaban el descontento social junto con la protesta (Pacceca, 2003; Constanzo, 2009). Buena parte de estas demandas o reclamos, fueron articulados a través del anarquismo que llevó adelante importantes huelgas, generando miedo y rechazo en la élite gobernante, cuya respuesta consistió en controlar estas acciones, a través de un repertorio represivo amplio, que en relación a la inmigración incluyó la formulación de dos leyes ${ }^{7}$ inspiradas en antecedentes de leyes francesas, estadounidenses o italianas (Constanzo, 2009). ${ }^{8}$

Por un lado, la ley de 1902, permitía la expulsión y prohibía el ingreso de todo extranjero que "haya sido condenado o sea perseguido por los tribunales extranjeros por crímenes o delitos comunes”, y de aquellos “cuya conducta comprometa la seguridad nacional o perturbe el orden público"9. Asimismo, señalaba que el extranjero contra el cual se emita una orden de expulsión, tenía hasta 3 días para abandonar el país y además autorizaba al Poder Ejecutivo a detenerlo hasta el momento del embarque como una "medida de seguridad pública”. Por otra parte, la ley de 1910, prohibía el ingreso a quienes "hayan sufrido condenas o estén condenados por delitos comunes que según las leyes argentinas merezcan pena corporal”, a los "anarquistas" y a los que hayan sido expulsados del país mientras no se derogue la orden de expulsión. Entre otras medidas, la ley prohibía las reuniones o asociaciones de personas que tuviesen como fin propagar doctrinas anarquistas, y establecía una serie de regulaciones para la realización de reuniones de asociaciones en la vía pública.

La relevancia que tienen estas leyes, radica en diferentes aspectos, entre los que se encuentra el ingreso, por primera vez en la normativa migratoria argentina de las nociones de "seguridad nacional” y "orden público” que en este caso hacían referencia a la amenaza representada por el anarquismo y los delitos que le eran atribuidos como la colocación de bombas y la realización de atentados (Scarzanella, 2003). La noción de expulsabilidad se vuelve muy relevante en este caso, ya que el castigo o la “solución” implementada por el estado adquiere esa impronta nacional a la que mereferíaanteriormente. Pues, si bien el problema para las élites gobernantes era ideológico (el “anarquismo”), se implementaba un trato diferenciado a los sujetos no-nacionales, así los inmigrantes, más allá de su efectiva expulsión, se convertían en sujetos expulsables, sobre todo cuando se trataba de cuestiones vinculadas a la seguridad y el crimen. En este sentido la (in)securitización de las migraciones implicó la inclusión de prácticas de detención y expulsión habilitadas por la normativa y ejecutadas a disposición del Ejecutivo Nacional por la Policía de la Capital. Las huelgas y protestas significaron desde las perspectivas de las élites una amenaza para su proyecto de inserción de la argentina en el mercado mundial. Asociando el anarquismo con un ideología importada por los inmigrantes italianos y españoles, las migraciones fueron crecientemente interpeladas y administradas como un problema de seguridad.

Como demuestran los registros de la época, las prácticas de expul- 
sión se materializaron a través de la Policía de la Capital. En un documento del Ministerio del Interior ${ }^{10}$, en el que se encuentran los registros de la fuerza de seguridad entre 1902 y 1914, consta la ejecución de 532 órdenes de expulsión de extranjeros de diversas nacionalidades, en mayor medida españoles e italianos, en el marco de la Ley $\mathrm{N}^{\circ}$ 4144. Sin embargo, es necesario aclarar que la detención y la expulsión como prácticasde control represivas no deben solapar el hecho de que durante este período se instrumentaron otras formas de control social ${ }^{11}$. Las elites gobernantes, durante el período que va de 1880-1920, desplegaron otros aparatos represivos y normalizadores, entre los que se destacan diferentes proyectos de modificación al código penal, como así también la modernización de la Policía de la capital, cuyo objetivo era garantizar que los individuos ocuparan los lugares que les eran asignados para asegurar el proyecto de desarrollo capitalista y de dominación política (Ruibal, 1993). En este sentido, el anarquismo formaba parte de un conjunto más vasto de figuras anormales o patológicas (como el delito, la vagancia, la mendicidad o la ebriedad) y de las denominadas clases peligrosas ${ }^{12}$ (Ruibal, 1993; Cf. Scarzanella, 2003).

Como ya advertí la Ley № 817 incorporaba la posibilidad de solicitar certificados, visados por las autoridades consulares o migratorias del país, en vistas de la selección de la inmigración. De este modo, durante la primera presidencia de Yrigoyen se dictaron dos decretos, que al solicitar un conjunto de certificados para poder ingresar como “inmigrante”, se constituían en la respuesta política del gobierno a los importantes prejuicios que había provocado la primera guerra mundial en la economía argentina y que tuvieron como resultado la agudización de los conflictos sociales (Devoto, 2001). Específicamente, se implementaron dos decretos en los que se "establecía que para poder inmigrar, además de la posesión de un pasaporte con foto, era preciso contar con tres certificados: de falta de antecedentes penales (librado por autoridad judicial, policial o comunal del país de origen), no mendicidad y de salud mental" (Devoto, 2001: 282). La introducción de estos certificados, incluyendo el de antecedentes penales, fue programada para aquellas personas que quisieran ingresar en el país en calidad de inmigrantes, pero no para todos los pasajeros que llegaban en los buques. Los temores en torno al orden social, se centraban sobre todo en los individuos que arribaban para permanecer y que además estaban conformados por las clases laboriosas, especialmente consideradas como clases peligrosas, y estrechamente vinculadas al crimen (Gayol y Kessler, 2002). De este modo, se iban construyendo e instrumentando los criterios que permitían al estado separar entre aquellos inmigrantes que serán caracterizados como deseables o indeseables (Quinteros, 2008).

El proceso de securitización implicó así la identificación del anarquismo como amenaza por parte de las élites, la intervención de las fuerzas policiales para el control de la inmigración a través del desarrollo de prácticas de detención y expulsión, colectivas e individuales, y la utilización de las técnicas de identificación. Aunque no todos los inmigrantes eran considerados como indeseables, la porción de ellos que se consideraba como amenaza propició la instalación de una sospecha generalizada sobre los 
individuos que llegaban al país en la categoría de inmigrantes. Pues para ingresar, permanecer y eventualmente obtener las "ventajas"13 concedidas por la normativa migratoria debían exponer si poseían antecedentes penales o no. Este tipo de medidas, orientadas a impedir el ingreso de aquellos sujetos que eran visualizados como una amenaza al orden social, expresaban con claridad los principios de visión y división del pensamiento de Estado, ya que las solicitudes de estos papeles sólo procedían para los sujetos considerados no nacionales, pero en particular a aquellos que ingresaban bajo la categoría de "inmigrantes". Así, los sujetos que viajaban en primera clase y que no se inscribían en esa categoría, aunque no nacionales de derecho, no se los consideraba como sujetos que representasen una amenaza para el orden social. Sin embargo, con el paso del tiempo, los controles migratorios y la solicitud de los antecedentes penales sería una cuestión cada vez más dependiente de la nacionalidad.

\section{De la sospecha sobre la "inmigración" a la de "todos los pasajeros"}

Años más tarde, la llegada de Alvear a la presidencia no supuso cambios importantes en la política migratoria, la idea de que el país necesitaba de la inmigración para desarrollarse sobrevivía junto con la preocupación por los reclamos políticos y las tensiones sociales, cuestión que se vio reflejada en el Reglamento Migratorio del 31 de diciembre de 1923 (Quinteros, 2008). Este decreto, que reglamentó por primera vez la Ley $N^{\circ} 817$ de 1876, sistematizó con claridad y detalle los motivos que impedían el ingreso al país. En concordancia con la normativa producida en los años previos se mantenía el criterio utilitarista, pues los motivos de prohibiciónpara el ingreso al territorio, mostraban que los migrantes tendrían que ser aptos para trabajar, al mismo tiempo que debían carecer de antecedentes penales. El decreto enumeraba una serie de características, a las que calificaba como "condiciones que impiden la entrada de pasajeros a la República”: para no quedar excluido era necesario de un certificado judicial o policial visado por cónsul argentino que compruebe que los individuos "no han estado bajo la acción de la justicia por delitos comunes o contra el orden social, durante los últimos cinco años”"14.

La creación de este reglamento trajo como consecuencia, diferentes interpretaciones en relación a quiénes eran los individuos a los que se podía prohibir el ingreso y solicitar los diferentes certificados, incluidos los antecedentes penales. La interpretación del reglamento era taxativa para DGI, los controles debían aplicarse no sólo a los individuos comprendidos en la categoría de inmigrantes sino a "todos los pasajeros". Empero, la presentación de recursos judiciales de habeas corpus por distintos extranjeros que fueron expulsados o reconducidos por la DGI, y que llegaron a la Corte Suprema de Justicia de la Nación ${ }^{15}$, muestran que en el camino de las instancias judiciales no siempre se le otorgaba la razón al organismo de aplicación de la normativa migratoria. Sin embargo, el máximo tribunal de 
justicia validó la interpretación de la DGI a través de diversos fallos, creando así una jurisprudencia que con el paso del tiempo será incorporada a la legislación migratoria. De este modo, las sospechas y preocupaciones que habían nacido alrededor de la inmigración, y en estrecha relación con la cuestión social, se ampliaron a todos los individuos que arribaran en los buques. Como consecuencias, los inmigrantes ya no eran el único objetivo de los controles migratorios, pues todos los extranjeros que quedaban por fuera de la categoría migratoria también debían cumplir con ciertos requisitos. El resultado fue que la categoría de “inmigración”, en tanto categoría de acceso a ventajas se mantuvo, mientras que perdía especificidad en relación a la seguridad, dicho de otro modo el control migratorio ampliaba su espectro hacia todas las movilidades de individuos no nacionales. Por otro lado, la disputa sobre la interpretación del alcance del control que se dirimió en la CSJN a favor de la DGI, muestra la relevancia de las prácticas administrativas y no sólo de los discursos, podría decirse que a veces las prácticas preceden a los discursos jurídicos.

La importancia de las burocracias también se puede observar en relación a las expulsiones realizadas por la Dirección General de Inmigración. Este organismo, se encontraba habilitado según la normativa vigente, por un lado, para expulsar a los individuos cuyas conductas se enmarcaban en la Ley de Residencia $\mathrm{N}^{\circ} 4144$, y por otro, para prohibir el ingreso y "reconducir" a los pasajeros que no cumplían con los requisitos solicitados por la ley. De modo similar a lo expuesto en el párrafo anterior, existió una disputa sobre si el ente administrativo podía expulsar a un individuo que ya había ingresado al territorio, y al que posteriormente se lo encontraba sin cumplir las condiciones de ingreso establecidas por la normativa. La revisión de algunos recursos de habeas corpus que también llegaron hasta la Corte Suprema ${ }^{16}$ de Justicia de la Nación, permite ver que el organismo de aplicación de la ley migratoria expulsaba a individuos que ya habían ingresado al país, y que si bien no cumplían con todos los requisitos de la normativa migratoria tampoco se encuadraban en la Ley de Residencia. Al igual que en el caso anterior, el organismo de aplicación creaba sus propias prácticas administrativas, con las que llenaba vacíos legales o puntos ambiguos de la legislación. Con el paso del tiempo estas interpretaciones constituidas en las prácticas serían avaladas por la jurisprudencia del máximo tribunal y ratificadaspor la legislación migratoria.

Ambos movimientos, el del control desplegado hacia todos los pasajeros y el de las expulsiones a los migrantes “clandestinos” nacen como el resultado de prácticas administrativas que se inscriben en espacios vacíos o ambiguos de la legislación, sólo posteriormente son avalados por la jurisprudencia y más tarde blanqueados por la normativa. Por otra parte, constituyen una transformación muy importante en el proceso de (in)securitización,ya que implican una ampliación de las medidas de control migratorio a partir de prácticas, que instrumentadas en un primer momento relación al anarquismo, son aplicadas posteriormente a todos los “extranjeros” que intentan ingresar al territorio. En este sentido, las prácticas producen no sólo una securitización de las migraciones sino de las diferentes 
movilidades que implican a sujetos no nacionales, la sospecha recae entonces no sólo en los individuos que se declaran como migrantes sino en todos los pasajeros que se trasladan en los barcos.

\section{Reflexiones finales}

La existencia de una abundante producción académica en relación a las políticas migratorias en Argentina desde diferentes disciplinas como la historia, la antropología y la sociología, no ha cubierto la relación entre seguridad y migración que aún se presenta como un terreno fértil para la investigación, ya sea que se aborde en la actualidad o en diferentes períodos históricos. En este artículo he presentado una forma de comprender el nexo entre seguridad y migración en las primeras décadas del siglo XX en Argentina, a partir de una selección y traducción de los aportes producidos recientemente a partir de la renovación del concepto de securitización, y la articulación con la noción de pensamiento de Estado propuesta por Sayad. Asimismo, también he recobrado bibliografía secundaria que me han servido para contextualizar el análisis. A lo largo del artículo, he reconsiderado la importancia de los discursos jurídicos, pero sobre todo de las prácticas administrativas, en la conversión de las migraciones y posteriormente las movilidades en un problema de seguridad. El análisis de tipo cualitativo se ha centrado en documentos como decretos, leyes, resoluciones, informes de organismos policiales y fallos judiciales de la Corte Suprema de Justicia de la Nación.

En relación a las prácticas administrativas y los discursos jurídicos o de las élites locales, he puesto de manifiesto, que las prácticas y técnicas utilizadas por los organismos de control migratorio por excelencia, ya sea la Policía de la Capital o la Dirección General de Inmigración, no fueron sencillamente el resultado del proceso de (in)securitización sino una posibilidad concreta para su despliegue y desarrollo. Sin las técnicas de identificación y la producción de los documentos de viaje como pasaportes y tarjetas de identidad, no se hubiese podido proceder a las prácticas de control de ingreso, “reconducción”o expulsión a la que se aludía en el discurso jurídico. Por otra parte, las prácticas de control y expulsión realizadas por la Dirección General de Inmigración, en particular la expansión hacia todos los tipos de movilidad y a los sujetos considerados como "clandestinos”, muestran cierta autonomía relativa del organismo cuyas prácticas amplían la (in)securitización y el universo de individuos pasibles de sospecha y control. En este sentido, mi interpretación sobre los discursos y prácticas tiende a dotarle mayor relevancia a las segundas a partir de los hallazgos que se producen a partir del análisis de los documentos empíricos.

Por otra parte, es necesario pensar si el proceso de (in)securitización de las migraciones no es en buena medida, resultado o subproducto del proceso de instauración del modelo capitalista agroexportador impulsado y sostenido por las élites locales y de los países centrales en el marco de la distribución internacional del trabajo. Por un lado, la necesidad de la inmi- 
gración que proveía de trabajo, y por otro, la acumulación de conflictos y tensiones sociales a lo largo de las últimas décadas del siglo XIX y las primeras del siglo XX, encontraron en el proceso de securitización un modo de regulación de las migraciones que permitía al mismo tiempo el ingreso y al permanencia de aquellos sujetos considerados necesarios para el desarrollo y la expulsión de aquellos individuos visualizados como una amenaza al orden nacional. Del mismo modo, permitía diferenciar a los inmigrantes, que accedían a derechos y ventajas, de aquellos sujetos peligrosos que por ser considerados improductivos se veían excluidos de los recursos distribuidos por el Estado.

Queda abierto un amplio campo de indagación en relación a las prácticas y técnicas administrativas y la circulación internacional de saberes e instituciones. Aunque hemos retomado algunos trabajos que van en ese sentido, este ámbito de preguntas se muestra como particularmente productivo. Los estudios que se preocupen por los procesos de (in)securitización deberían tener en cuenta, al analizar las prácticas realizadas por los organismos de control, que las mismas pueden configurar parte de un entramado más complejo que se construye a partir de la importación y exportación de saberes, prácticas e instituciones de otros contextos nacionales. Aunque ya existen algunos antecedentes, aún queda un buen camino por recorrer en relación a la producción de los documentos de viaje, el surgimiento de las técnicas de identificación o la colaboración entre organismos de control migratorio, no sólo a principios del siglo XX sino también en la actualidad. Estudios que sigan esta dirección podrían ayudarnos a comprender en qué medida la securitización ha sido construida de un modo transnacional o internacional y cuáles son los efectos que esto implica en los discursos y prácticas.

Por último, quiero destacar que aún existenimportantes dimensiones, en relación al nexo entre seguridad y migración, que permanecen inexploradas. El estudio de otros períodos históricos podría ayudar a comprender cómo se constituyen estos procesos en el largo plazo, mientras que el abordaje de nuevos casos permitiría el desarrollo y consolidación de investigaciones que aborden la circulación internacional de saberes e instituciones, tal como explique en el párrafo anterior. El estudio de esta temática en otros países de América Latina se revela como un espacio fértil para la producción de nuevos puntos de vista o enfoques en relación a la securitización de las migraciones. Asimismo, debería constituirse en un primer paso para la realización de estudios comparativos con otros países y regiones, tales como Estados Unidos y Europa, donde la relación entre seguridad y migración ha sido ampliamente abordada desde diferentes disciplinas y enfoques teóricos. Los intercambios entre los estudios de diferentes regiones y países podrían contribuir a la producción de nuevos interrogantes y explicaciones sobre la aludida relación. 


\section{Notas}

${ }^{1}$ El presente artículo se enmarca en el proyecto de investigación "Migración y políticas de control: prácticas institucionales y experiencias sociales", subsidiado por la Secretaría de Ciencia y Tecnología de la Universidad Nacional de Córdoba, Argentina. Período 2014-2015.

${ }^{2}$ A lo largo del texto utilizaré de modo indistinto las palabras securitización o (in)securitización para referirme al mismo proceso.

${ }^{3}$ Para profundizar en el campo de los estudios críticos de la seguridad y las escuelas de Copenhague, Paris y Aberystwyth ver (Waever, 2004; Bigo, 2008).

${ }^{4}$ La noción de doble pena explicitada por Sayad, implica que los inmigrantes no sólo pueden ser juzgados por un delito en particular, sino que la migración es considerada en sí misma como un delito. En sus propias palabras:"En este caso particular, la delincuencia no es solamente la de los delitos que la policía tiene que conocer, la de los delitos que registran las estadísticas de la criminalidad, sino, que es una delincuencia que oculta otra, es una delincuencia que se diría de situación estatutaria (casi 'ontológica'), pues se confunde, en lo más profundo de nuestro modo de pensamiento (i.e., el pensamiento de Estado), con la existencia misma del inmigrado y con el hecho mismo de la inmigración” (Sayad, 2010: 390).

${ }^{5}$ La noción de expulsabilidad puede ser leída como una de las marcas del pensamiento de Estado, pues como afirma el autor "está en la naturaleza misma de la soberanía de la nación expulsar a quien le parezca de los residentes extranjeros (en el sentido de la nacionalidad) y está en la naturaleza del extranjero (nacionalmente hablando) ser expulsable, y poco importa, pues, que sea efectivamente expulsado o no" (Sayad, 2010: 403). De modo más categórico, explica que la expulsabilidad y el pensamiento de Estado son lo mismo, ya que más allá de la expulsión efectiva "la expulsabilidad del extranjero es el signo por excelencia de una de las prerrogativas esenciales de la soberanía nacional, y es ella también la marca del pensamiento de Estado, por no decir que es también el pensamiento de Estado en sí mismo” (Ibíd.)

${ }^{6}$ De acuerdo con el artículo 12 de la Ley $\mathrm{N}^{\circ} 817$ se definía como inmigrante a "todo extranjero jornalero, artesano, industrial, agricultor o profesor, que siendo menor de sesenta años y acreditando su moralidad y sus aptitudes, llegase a la República para establecerse en ella, en buques a vapor o a vela, pagando pasaje de segunda o tercera clase, o teniendo el viaje pagado por cuenta de la Nación, de las Provincias o de las empresas particulares protectoras de la inmigración y la colonización”

${ }^{7}$ Ley de Residencia de Extranjeros $\mathrm{N}^{\circ} 4144$ de 1902 y la Ley de Defensa Social $\mathrm{N}^{\circ}$ 7902 de 1910.

${ }^{8}$ La alusión de Constanzo (2009) sobre la inspiración de las leyes argentinas en la de otros países, nos lleva a advertir que las políticas migratorias, o más sencillamente ciertas medidas que se aplican, deben ser observadas en un contexto que trascienda los límites del Estado-nación.

${ }^{9}$ Ley $\mathrm{N}^{\circ} 4144$ de 1902. 
${ }^{10}$ Ministerio del Interior, $1^{\circ}$ Sección. Copiador Anarquismo. Archivo General de la Nación.

${ }^{11}$ Es oportuno señalar que futuras investigaciones deberían profundizar temas tales como la medicalización de la homosexualidad, su relación con el control social y en particular con el control migratorio. Aunque esta temática no se constituye como objeto de este artículo, se sugiere consultar a Salessi (1995) y Guy (1992). Por otro lado, se sugiere ver el trabajo de Di Liscia y Fernández Marrón (2009) en el que analizan las políticas de exclusión dirigidas hacia inmigrantes con tracoma en las primeras décadas del silgo XX.

${ }^{12}$ La idea de "clases peligrosas", hace referencia a "aquellos individuos que no pertenecen ni a la categoría de los normales ni a la de los anormales, pero que constituyen una clase peligrosa por el ambiente en que viven. Estos sujetos son aquellos que ya han cometido crímenes, pero también los que por su forma de vivir podrían cometerlos como el caso de los vagos y los ebrios habituales- y finalmente los individuos que manifiestan una predisposición natural a cometer delitos, como los anormales y los defectuosos morales” (Ruibal, 1993: 11).

${ }^{13}$ Estas ventajas incluían el alojamiento en el hotel de inmigrantes, los traslados hacia el interior del país y algunas franquicias para el ingreso de herramientas vinculadas a las actividades laborales. Ver Ley $\mathrm{N}^{\circ} 817$.

${ }^{14}$ Decreto del 31 de diciembre de 1923.

${ }^{15} \mathrm{El}$ alcance de las medidas de control tuvo diferentes interpretaciones, los actores judiciales y la Dirección General de Inmigración no siempre compartían el mismo criterio en relación a si las medidas deberían ser aplicadas a los "inmigrantes" o a "todos los pasajeros". Como se puede a través de algunas sentenciasjudiciales, el Departamento General de Inmigración, interpretaba que los impedimentos establecidos en el decreto de diciembre de 1923 regían no sólo para los inmigrantes sino para todos los pasajeros. Aunque a veces las diferentes instancias judiciales no coincidían, la Corte Suprema de Justicia de la Nación a través de dos sentencias,la del caso Irene Amor Magaz de González del 8 junio de 1927 (Fallos 148: 420); y el caso Francisco Macciá y Ventura Gassol con sentencia del 16 mayo de 1928 (Fallos 151: 211) terminó por reconocer como válida la postura del DGI. Para ver las interpretaciones que ha realizado la CSJN en relación a la noción de habitante e inmigrante ver Begala (2007).

${ }^{16}$ La referencia es a las sentencias de la Corte Suprema de Justicia de la Nación: el caso de Jorge Pasaradelis con sentencia del 15 de septiembre de 1934 (Fallos 171:317) y Kaiser Teodoro con sentencia del 3 de mayo de 1939 (Fallos 183:373). Allí la Corte indica que quienes ingresan de modo "clandestino" no pueden ser considerados habitantes ni permanecer en el territorio, habilitando la expulsión de los mismos. 
Polis, Revista Latinoamericana, Volumen 15, No 44, 2016

\section{Bibliografía}

Begala, Silvana (2007), "El concepto de habitante, una medida al reconocimiento y al ejercicio de los Derechos de los extranjeros”, en Anuario, $N^{\circ} 10$.

Biernat, Carolina (2007), ¿Buenos o útiles? La política inmigratoria del peronismo, Biblos, Buenos Aires.

Bigo, Didier (2002),"Security and Inmigration: Toward a Critique of the Governmentality of Unease”, en Alternatives, $\mathrm{N}^{\circ} 27$.

Ídem (2008), “International political sociology”, en Willias, Paul Ed.; Security Studies: an introduction, Routledge, Oxon.

Bigo, Didier y Tsoukala, Anastassia (2008), "Understanding (in)security”, en Bigo, Didier y Tsoukala, Anastassia, eds.; Terror, Insecurity and Liberty, Routledge, Oxon.

Constanzo, Gabriela (2009), Los indeseables: las leyes de residencia y defensa social, Madreselva, Buenos Aires.

Bourdieu, Pierre (1997), Razones prácticas. Sobre la teoría de la acción, Anagrama, Barcelona.

Devoto, Fernando (2001), "El revés de la trama: Políticas migratorias y prácticas administrativas en la argentina (1919-1949)”, en Desarrollo Económico, Vol, 41. ํ 162.

Ídem (2009), Historia de la Inmigración en la Argentina, Sudamericana, Buenos Aires.

Di Liscia, María y Fernández Marrón, Melisa (2009), “Sin puerto para el sueño americano. Políticas de exclusión, migración y tracoma en Argentina (1908-1930)", en Revista Nuevo Mundo, [en línea: https:// nuevomundo.revues.org/57786 Visitada en Mayo, 2016]

Domenech, Eduardo (2011), “Crónica de una 'amenaza' anunciada. Inmigración e 'ilegalidad': visiones de Estado en la Argentina contemporánea”, en Feldman-Bianco, Bela; Villa, Marta; Rivera, Liliana; Stefoni, Carolina (comps), La construcción social del sujeto migrante en América Latina: prácticas, representaciones y categorías, CLACSO / FLACSO-Ecuador / UAH,Quito.

Fallos, (2015), Fallos de la Corte Suprema de Justicia de la Nación, [en línea: http://sj.csjn.gov.ar/sj/tomosFallos.do?method=iniciar Visitada en Diciembre, 2015]

Galeano, Diego (2009), "Las conferencias sudamericanas de policías y la problemática de los ‘delincuentes viajeros', 1905-1920”, en Bohoslavsky, 
Ernesto, Caimari, Lila y Schettini, Cristiana org.; La policía en perspectiva histórica. Argentina y Brasil (del siglo XIX a la actualidad), CD-Rom, Buenos Aires.

García Ferrari, Mercedes (2010),Ladrones conocidos / Sospechosos reservados. Identificación policial en Buenos Aires, 1850-1905, Prometeo, Buenos Aires.

Ídem (2015), Marcas de identidad. Juan Vucetich y el surgimiento transnacional de la dactiloscopia (1888-1913), Prohistoria, Rosario.

Gayol, Sandra y Kessler, Gabriel (2002). “Introducción”, en Gayol, Sandra y Kessler, Gabriel comps.; Violencias, delitos y justicias en la Argentina, Manantial, Buenos Aires.

GEADIS (2002), “De inmigrantes a delincuentes. La producción de los indocumentados como amenaza social en el discurso policial”, en Cuadernos de Antropología social, N¹5.

Guy, Donna (1992), “White slavery,' citizenship and nationality in Argentina”, en Andrew, Parker; Mary, Russo; Doris, Somer; Patricia Yaegereds; Nationalism and sexualities, Routledge, New York.

Huysmans, Jef (2006), The politics of insecurity. Fear, migration and asylum in the EU,Routledge, Oxon.

Huysmans, Jef y Squire, Viqui (2009), “Migration and security”, en Dunn Caveltty, Myriam y Mauer, Victor eds.; Handbook of Security Studies, Routledge, London.

Novick, Susana (1997), “Políticas inmigratorias en la Argentina”, en Oteiza, Enrique; Novick, Susana; Aruj, Roberto; Inmigración y Discriminación. Políticas y Discursos, Grupo Editor Universitario, Buenos Aires.

Pacecca, María Inés (2000), “Legislación, migración limítrofe y vulnerabilidad social”, en Realidad Económica, № 171.

Ídem (2003), “El fantasma en la máquina: la praxis política de los extranjeros y la ley de Residencia”, en Susana Villavicencio (Ed), Contornos de la ciudadanía. Nativos y extranjeros en el centenario, Eudeba, Buenos Aires.

Pacecca, María Inés; Courtis, Corina; Pita María Victoria (2009), “Migración y seguridad en la Argentina. Construcciones discursivas y prácticas”, Miemo.

Quinteros, Marcela (2008), Os olhos da na?ão. As imagens construídas sobre o estrangeiro nas políticas inmigratorias argentinas (1930-1955), Instituto Memoria, Curitiba. 
Polis, Revista Latinoamericana, Volumen 15, No 44, 2016

Ruibal, Celina Beatriz (1993), Ideología del control social. Buenos Aires 1880-1920, CEAL, Buenos Aires.

Salessi, Jorge (1995), “The Argentine Dissemination of Homosexuality, 1890-1914”, en Emilie, Bergmann y Paul Julian, Smith eds.; Entiendes? Queer Readings, Hispanic Writings, Duke University Press, Durham.

Sayad, Abdelmalek (2008), “Estado, nación e inmigración. El orden nacional ante el desafío de la inmigración”, en Apuntes de investigación del CECYP, $\mathrm{N}^{\circ} 13$.

Ídem (2010), La doble ausencia. De las ilusiones del emigrado a los padecimientos del inmigrado, Anthropos, Barcelona.

Scarzanella, Eugenia, (2003), Ni gringos ni indios. Inmigración, criminalidad y racismo en la Argentina 1890-1949, Universidad Nacional de Quilmes, Buenos Aires.

Tiscornia, Sofía, (2004a), “Entre el honor y los parientes. Los edictos policiales y los Fallos de la Corte Suprema de Justicia. El caso de 'las Damas de la Calle Florida’ (1948-1957)”, en Tiscornia, Sofía comp.; Burocracias y violencia. Estudios de antropología jurídica, Antropografía, Buenos Aires.

Ídem (2004b), “Entre el imperio del "Estado de policía” y los límites del derecho. Seguridad ciudadana y policía en argentina”, en Nueva Sociedad, $\mathrm{N}^{\circ}$ 191.

Torpey, John (2000), The invetion of passport. Survillance, Citizenship and State, Cambrige University Press, Cambridge.

Weaver, Ole; Buzan, Barry; Klestrup, Mortenet.al (1993), Identity, migration and the new security agenda in Europe, St. Martin's, New York.

Waever, Ole (1998), “Securitizatioin and desecuritization”, en Lipschutz, Ronnie ed.; On Security, Columbia University Press, New York.

Ídem (2004), “Aberystwyth, Paris, Copenhagen New 'Schools’ in Security. Theory and their Origins between Core and Periphery”, trabajo presentado en la reunión anual de la International Studies Association, Montreal. 